\section{The therapeutic potential of rhEPO in multiple sclerosis and Friedreich's ataxia}

Recombinant human erythropoietin (rhEPO) has been a focus of investigation in neurodegenerative diseases since the unexpected finding that it has broad neuroprotective and cardioprotective capabilities. Two recent studies have highlighted the potential usefulness of this agent in chronic progressive multiple sclerosis (MS) and Friedreich's ataxia (FA).

Ehrenreich et al., encouraged by results obtained with rhEPO in experimental models and first clinical studies in other neurodegenerative diseases, devised an exploratory open-label phase Ila study in patients with chronic progressive MS. Five patients with MS and two drug-naive patients with Parkinson's disease (controls) received 48,000 IU of rhEPO, and three patients with MS received $8,000 \mathrm{IU}$ of rhEPO. After a lead-in phase of 46-weeks, patients were started on 12 weeks of treatment with weekly rhEPO, followed by 12 weeks of fortnightly rhEPO, then 24 weeks of post-treatment assessment.

All five MS patients who were given the high dose of rhEPO showed marked improvements in motor function; maximum walking distance improved significantly $(P \leq 0.002)$, resulting in reduced expanded disability status scale (EDSS) score, and central motor conduction time also decreased. This treatment group also had improved performance in several measures of executive functioning. All these improvements persisted for 3-6 months after treatment had ended. By contrast, no improvements in these measures were noted in the patients receiving the lower dose of rhEPO, or in controls. No adverse events occurred during follow-up, demonstrating the safety of rhEPO, even at high doses.

FA is caused by a GAA-trinucleotide expansion in the frataxin gene $(F X N)$, which reduces expression of the mitochondrial protein frataxin. Boesch et al. performed an investigation to follow up their recent observation that rhEPO increases frataxin levels in lymphocytes isolated from FA patients. In an open-label clinical pilot study, 12 patients with FA were given 5,000IU rhEPO three times weekly. Frataxin levels measured in isolated lymphocytes by enzymelinked immunosorbent assay were found to be significantly increased after 2 and 8 weeks of treatment (mean change $+27 \%$, range $15-$ $63 \% ; P<0.01$ vs baseline); enhanced frataxin was maintained over the 8 weeks of treatment. All patients also showed a reduction of urinary 8-hydroxydeoxyguanosine (mean $21.6 \pm 11.2$ vs $10.9 \pm 2.3 \mathrm{ng} / \mathrm{mg}$ creatinine; $P<0.02$ for baseline vs week 8 ) and serum peroxides (mean $162.1 \pm 171.0$ vs $128.7 \pm 162.4 \mu \mathrm{m} ; P=0.39$ for baseline vs week 8), both of which are important markers of oxidative stress. Some improvement was noted in the severity of ataxia, most especially in patients with increased frataxin levels, who experienced a mean $11.5 \%$ reduction in SARA (Scale for the Assessment and Rating of Ataxia) points.

Following the encouraging findings from both studies, the authors advocate further investigations into the effects of rhEPO. Boesch et al. urge that the potency of rhEPO is assessed over a longer period. Similarly, Ehrenreich et al. recommend a double-blind, placebo-controlled proof-of-concept trial on high-dose $(48,000 \mathrm{IU}$ weekly) EPO treatment in chronic progressive MS that continues for at least 3 years and involves at least two EPO treatment cycles.

Original articles Ehrenreich $\mathrm{H}$ et al. (2007) Exploring recombinant human erythropoietin in chronic progressive multiple sclerosis. Brain 130: 2577-2588

Boesch S et al. (2007) Friedreich's ataxia: clinical pilot trial with recombinant human erythropoietin. Ann Neurol [doi:10.1002/ana.21177]

\section{High-dose idebenone to improve neurological function in Friedreich's ataxia}

Friedreich's ataxia (FA) causes neurological impairment, cardiomyopathy, scoliosis and diabetes. Many FA symptoms have been attributed to oxidative tissue damage, and the potential for antioxidant therapy is currently being explored. Idebenone, a potent antioxidant, reduces the level of oxidative markers in the blood and improves cardiac hypertrophy in patients with FA at a dose of $5 \mathrm{mg} / \mathrm{kg}$, but no beneficial effect on neurological function has been apparent at this dose. Higher doses given early in the disease course, however, might prove more successful.

Di Prospero et al. examined this possibility in 48 young patients with FA (aged 9-17 years). The patients were divided into four groups and given placebo, or idebenone at approximately 